\title{
Low burden atrial fibrillation and iron deficiency anemia as a contributing factor of thromboembolic events
}

Keywords: atrial fibrillation, iron deficiency anemia, atrial fibrillation, atherothrombosis, cardiogenic embolism, arterial dissection, antiphospholipid antibody syndrome, vasculitis syndrome

Abbreviations: AF, atrial fibrillation; IDA, iron deficiency anemia; USG, ultrasonography; $\mathrm{MCH}$, mean corpuscular hemoglobin; PRBCs, packed red blood cells

\section{Introduction}

Atrial fibrillation (AF) is unequivocally a certain factor of thromboembolic events. Iron deficiency anemia has not been extensively studied as a cause of stroke in adults. In contrast, iron deficiency anemia has been several times described as a cause of stroke in infants and children. ${ }^{1-3}$ In adults, stroke and peripheral arterial ischemia has been attributed to thrombotic, embolic, or hemodynamic events and common causes include atrial fibrillation, atherothrombosis, cardiogenic embolism, arterial dissection, antiphospholipid antibody syndrome, vasculitis syndrome etc. ${ }^{4}$ Although the association between anemia and acute ischemic stroke has been reported, anemia is not well recognized as a potential cause of stroke and peripheral ischemia. We herein report one case of two serial events: peripheral ischemia and stroke in an elderly man that was caused by AF in conjunction with iron deficiency anemia (IDA).

\section{Case report}

A 72-year-old male patient was admitted to our hospital with numbness, pain, coldness and pallor of his right forearm and hand that begun approximately one hour ago and persisted. He complained of ache and intense pain thereafter and noticed pallor at the fingers and palm of his right hand. His past medical history included cholecystectomy, appendectomy, benign prostate hypertrophy, choroidal polypoidal vasculopathy, and cataract bilaterally. He smokes and his family history was negative for neurological or hematologic diseases. Three years ago, mild anemia appeared (Hct: 38\%, MCV: $75 \mathrm{Fl}$ ), which was attributed to iron deficiency. The patient underwent endoscopic evaluation with gastroscopy, colonoscopy and MRI enterography, but no bleeding site was revealed. He was suggested to take orally iron replacement but did not comply due to gastrointestinal discomfort. One month ago, an episode of atrial fibrillation was recorded, incidentally by his attending pneumonologist on clinical examination. It was confirmed by HCG, lasted a few minutes and it was terminated spontaneously.

An urgent artery doppler ultrasonography (USG) of his upper extremities revealed the presence of an acute thrombus at his right ulnar artery in the cubital fossa. His general physical examination revealed pallor and coldness in the right hand with absent pulse in the ulnar artery at the time of his presentation at the ED. The pulse was restored after one hour and the pallor and coldness disappeared. The rest of the detailed examination was normal. Angiosurgery
Volume 14 Issue 3 - 202I

\author{
Vassilara Foula,' Papadopoulos loannis,' \\ Pipilis Athanasios, ${ }^{2}$ Plomaritoglou Androniki, ${ }^{3}$ \\ Pothitos George,' Spiridaki Aikaterini' \\ 'Department of Internal Medicine, Hygeia Hospital, Greece \\ ${ }^{2}$ Department of Cardiology, Hygeia Hospital, Greece \\ ${ }^{3}$ Department of Neurology, Hygeia Hospital, Greece
}

Correspondence: Vassilara Foula, Department of Internal Medicine, Hygeia Hospital, Athens, Greece, Tel +3069324I5289, Email fvassilara@hygeia.grs

Received: May 31, 202I | Published: June 15, 202 I

consultation suggested low molecular weight heparin according to his weight and he received enoxaparin sodium $80 \mathrm{mg} \mathrm{SC} \mathrm{q12hr}$. The ECG was on sinus rhythm, X-ray normal and the transthoracic echocardiogram bubble study showed no evidence of septal defects or cardiac source of thrombus.

Laboratory analysis displayed an iron-deficiency anemia with a hemoglobin level of $8.0 \mathrm{~g} / \mathrm{dL}(13-17)$, hematocrit of $27 \%$, a mean corpuscular volume (MCV) of 75.0fL (80-92), a mean corpuscular hemoglobin $(\mathrm{MCH})$ level of 22.5pg (27-31), and a platelet count of $270 \times 10 \mathrm{E} 3 / \mu \mathrm{L}(130-400)$. His serum ferritin level was $8 \mathrm{ng} / \mathrm{mL}$ (7-26). The other laboratory parameters including white blood count, total cholesterol 204mg/dl, LDL 100mg/dl, HDL 67mg/dl, TG 154mg/ $\mathrm{dl}$ chemistry panel, urea, creatinine, were also within normal limits.

The patient received iron replacement therapy intravenously with ferric carboxymaltose $1000 \mathrm{mg}$ and was transfused with one unit of packed red blood cells (PRBCs). Holter ECG demonstrated a noticeably short episode of hyperventricular tachycardia lasted 4.3 sec. Given the short duration of the episode an atrial fibrillation event could not be safely excluded.

In order to clear out the cause of the patient's iron deficiency we investigated the gastrointestinal tract. Upper and lower gastrointestinal endoscopy did not find abnormalities and capsule endoscopy ruled out possible bleeding sites at the small intestine.

Thrombophilia studies were negative following tests for antiphospholipid antibodies (anticardiolipin IgA, IgG, and IgM antibodies), and no evidence of lupus anticoagulant was detected. Anti $\beta 2$ GPI IgM, IgG negative, IgA positive in a titer $32 \mathrm{U} / \mathrm{ml}$ (normal up to 20). Protein C, protein S, antithrombin III, fibrinogen, vitamin B12, folate, homocysteine levels, transglutaminase antibodies, prothrombin and partial prothrombin levels were within normal ranges, and the prothrombin gene variant G20210 and Factor V Leiden mutations were not detected. 
A transesophageal echocardiography was performed and no clots in the left atrium, PFO or septal defects were detected. Calcified plaques in the descending thoracic artery and in the left coronary artery were depicted. At the $6^{\text {th }}$ day of his hospitalization and while the patient continued anticoagulation therapy with low molecular weight heparin and anti-Xa assay was into therapeutic levels, he suddenly developed Broca's aphasia. His symptoms improved gradually and lasted $<24$ hour.

Brain MRI showed small lesions (diameter $<5 \mathrm{~mm}$ ) of increased signal on T2 and FLAIR sequences, with characteristics suggesting recent ischemic lesions, at cortex of the posterior left parietal lobe as well as at the cortex and the subcortical white matter of the right parietal lobe. Rosuvastatin $20 \mathrm{mg}$ was added to his regimen.

Further evaluation with $\mathrm{CT}$ angiography of aortic arc, extracranial and intracranial arteries revealed calcified atheromatous plaques at the origin of the right and left internal carotid artery without significant stenosis or the presence of ulcerative plaques. CT pulmonary angiography was negative for emboli and Doppler ultrasonography of lower limb veins was negative for acute or old thrombotic events, both at deep and superficial veins. CT scan of thorax and abdomen didn't show radiological findings of underlying malignancy.

The patient recovered completely with no neurologic deficits. His anticoagulation therapy was overlapped with vitamin $\mathrm{K}$ antagonist acenocoumarol, while awaiting the results for thrombophilia but the treatment switched to apixaban $5 \mathrm{mg}$ bid, when the results turned out to be negative. The patient was discharged from the hospital with improved hematocrit (Hct:35,7\%).

\section{Discussion}

The risk of stroke in patients with $\mathrm{AF}$ is not homogeneous and several clinical factors can contribute to it. The development of $\mathrm{CHADS}_{2}$ and more recently $\mathrm{CHA}_{2} \mathrm{DS}_{2}$-VASc scores to calculate which patients are candidates for anticoagulation may underestimate the risk. Genetic and acquired factors for hypercoagulable states do not incorporate into the score. From the hematological point of view there is a lack of recommendation. The majority of studies come from cardiovascular and brain units departments. Specifically, the patient according to $\mathrm{CHA}_{2} \mathrm{DS}_{2}$-VASc score had an estimated risk for stroke $0.6 \%$ per year as he had $\mathrm{CHA}_{2} \mathrm{DS}_{2}$-VASc score 1 . The option not to give anticoagulants at the first presentation of $\mathrm{AF}$ was in accordance with the last guidelines of American Heart Association which suggest to give anticoagulation therapy to all patients with $\mathrm{CHA}_{2} \mathrm{DS}_{2}-\mathrm{VASc}$ score $>1$ although not in accordance with the European guidelines, which suggest anticoagulation therapy to all men patients with $\mathrm{CHA}_{2} \mathrm{DS}_{2}$-VASc score $\geq 1 . .^{15,16}$ In the real clinical practice the strict implementation of guidelines fail to protect a minority of patients or to over treat some others. The decision for treating a patient becomes even more difficult when the results from different studies are not always in consistency.

The association between iron deficiency anemia (IDA) and ischemic stroke has been studied more frequently in children than in

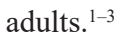

Multiple pathophysiological mechanisms have been implied in the development of embolic phenomena in patients with IDA including thrombocytosis, hypercoagulable state, and anemic hypoxia. ${ }^{5}$ The putative mechanisms underlying anemia and stroke syndromes are not completely understood, and it is believed that iron deficiency, may cause ischemic stroke by several potential mechanisms. Microcytic red blood cells in IDA, have altered deformability, which increases the blood viscosity and may increase the risk of venous thrombosis..$^{5-7}$ Furthermore, the decreased oxygen-carrying capacity of the erythrocytes results in anemic hypoxia, and anemic patients need increased blood flow to the brain and peripheral tissues to compensate for the lack of oxygen in the blood. ${ }^{8}$ This increased blood flow can cause vascular endothelial damage, favoring thrombus formation especially in the setting of an underlying atherosclerotic disease that is often present in elderly patients. ${ }^{9,10}$ Low iron levels alter platelets activity contributing to increased aggregation and induce the production of erythropoietin, which stimulates vasoconstriction dependent hypertension and angiogenesis.

When trying to identify and categorize all stroke events, stroke of undetermined etiology is included. ${ }^{4}$ In our case two ischemic events occurred abruptly to our patient and despite of the detailed evaluation, no obvious thromboembolic source was revealed. Transesophageal echocardiography was negative for clots formation.

Cardiovascular events are a well-recognized source for embolic occurrences. The concern is that asymptomatic and/or undetected recurrences of atrial fibrillation could expose patients to increase risks of ischemic stroke and thromboembolic events. ${ }^{11,12}$ In our case two ischemic episodes happened, but serial ECG showed sinus rhythm and the 24hours Holter ECG displayed only a short duration episode of supraventricular arrhythmia or AF that lasted 4,3sec. In a recent study, atrial fibrillation was defined as an episode of irregular heart rhythm, without detectable P waves, lasting more than 30seconds. ${ }^{13}$

The total number of AF episodes did not correlate with stroke risk, suggesting that episode duration rather than frequency was the more critical component of AF burden. It is thought that the risk of stroke is time-dependent in patients with $\mathrm{AF} .{ }^{14-15,17}$

Selection of antithrombotic therapy should be based on the risk of thromboembolism irrespective of whether the AF pattern is paroxysmal, persistent, or permanent regarding $\mathrm{CHA}_{2} \mathrm{DS}_{2}-\mathrm{VASc}$ score. ${ }^{16}$ In a Greek, country - wide registry of AF, it was proved that paroxysmal AF was treated less intensively than permanent $\mathrm{AF}$, contrary to existing guidelines. ${ }^{18}$

Asymptomatic atrial tachyarrhythmias, are detected frequently in patients with implantable devices and were associated with a significantly increased risk of ischemic stroke or systemic embolism. The minimum duration is considered more than 6 mins. ${ }^{17}$ At the same study the authors did not analyze device-detected events of 6 minutes or less, which occurred frequently and which might be clinically important. This implication could apply to our patient. However, further studies are needed to prove this hypothesis.

It is overly simplistic to consider that AF is the only cause of thrombus formation. Co-existence with aortic plaques, left atrial dysfunction, akinetic left ventricular segment are contributing factors to stroke events. ${ }^{15,19}$

There are more than 200 known causes of ischemic stroke; some of them can coexist with AF. On the other hand in some patients with infrequent $\mathrm{AF}$, the arrhythmia is not causually related to the stroke and another cofounding factor might be the underlying cause. ${ }^{20}$

It is not clear what the precise duration of $\mathrm{AF}$ associated with stroke is and further studies are needed to determine it. Current guidelines suggest performing 24 or more hours of ECG monitoring to rule out atrial fibrillation in patients with an ischemic stroke, but the most effective duration of monitoring has not been determined. ${ }^{14}$ The use of additional ECG monitoring beyond 24hours after cryptogenic stroke might detect further atrial fibrillation events, but it is currently left to the physician to decide. ${ }^{15}$ 
For patients with low-burden AF, denoting duration of AF less than $5 \mathrm{~min}$ such as in our patient, multicenter prospective studies are needed to clarify if anticoagulation can be safely started or stopped based on AF burden. ${ }^{21}$

We think that in our patient iron deficiency anemia was a concomitant potential factor in addition to $\mathrm{AF}$ in thrombus formation. We think that the role of iron deficiency anemia has been underestimated in the stroke events. In our case even though the thrombus was visible in the ulnar artery we could not identify the site of thrombus formation. Furthermore, despite the anticoagulation treatment which was in therapeutic levels according to antiXa levels the patient presented on the $6^{\text {th }}$ day of hospitalization with new thromboebolic events in the brain circulation, which confirms previous publications that some patients present with thromboebolic events despite the anticoagulation treatment. If a formation of new thrombus or a non-visualized, nondissolved remnant thrombus was the cause of new emboli remained questionable.

Our patient experienced an ischemic stroke, in spite of being treated with low molecular weight heparin, suggesting that anemia and iron deficiency of our patient might aggravate the two embolic episodes via ethothelial damage in the presence of calcified plaques, in his large arteries.

\section{Conclusion}

In patients with acute ischemic stroke and anemia, it is important to consider iron deficiency anemia as additional cause of stroke in order to prevent stroke recurrence. ${ }^{22}$ Despite our case report, the reason why few patients with iron deficiency anemia result in thromboembolic events is not clear. It might be hypothesized that iron deficiency anemia intensifies possible unknown/subclinical causes. Therefore, further studies of similar cases are necessary to understand the underlying etiology.

\section{Acknowledgments}

None.

\section{Conflicts of interest}

The rest of the authors declare do not have conflicts of interest.

\section{Funding}

This manuscript was not supported by any funding.

\section{References}

1. Azab SF, Abdelsalam SM, Saleh SH, et al. Iron deficiency anemia as a risk factor for cerebrovascular events in early childhood: a case-control study. Ann Hematol. 2014;93(4):571-576.

2. Young RS, Rannels DE, Hilmo A, et al. Severe anemia in childhood presenting as transient ischemic attacks. Stroke. 1983;14(4):622-623.

3. Hartfield DS, Lowry NJ, Keene DL, et al. Iron deficiency: a cause of stroke in infants and children. Pediatr Neurol. 1997;16(1):50-53.

4. Adams Jr HP, del Zoppo G, Alberts MJ, et al. Guidelines for the early management of adults with ischemic stroke: a guideline from the American Heart Association/American Stroke Association Stroke Council, Clinical Cardiology Council, Cardiovascular Radiology and Intervention Council, and the Atherosclerotic Peripheral Vascular Disease and Quality of Care Outcomes in Research Interdisciplinary Working Groups: the American Academy of Neurology affirms the value of this guideline as an educational tool for neurologists. Circulation. 2007;115(20):e478-534.
5. Ogata T, Kamouchi M, Kitazono T, et al. Cerebral venous thrombosis associated with iron deficiency anemia. J Stroke Cerebrovasc Dis. 2008;17(6):426-428.

6. Franchini M, Targher G, Montagnana M, et al. Iron and thrombosis. Ann Hematol. 2008;87(3):167-173.

7. Swann IL, Kendra JR. Severe iron deficiency anemia and stroke. Clin Lab Haematol. 2000;22(4):221-223.

8. Batur Caglayan HZ, Nazliel B, Irkec C, et al. Iron-Deficiency Anemia Leading to Transient Ischemic Attacks due to Intraluminal Carotid Artery Thrombus. Case Rep Neurol Med. 2013;2013:813415.

9. Mehta PJ, Chapman S, Jayam-Trouth A, et al. Acute ischemic stroke secondary to iron deficiency anemia: a Case Report. Case Rep Neurol Med. 2012;2012:487080.

10. Naito H, Naka H, Kanaya Y, et al. Two cases of acute ischemic stroke associated with iron deficiency anemia due to bleeding from uterine fibroids in middle-aged women. Intern Med. 2014;53(21):2533-2537.

11. Khan M, Miller DJ. Detection of paroxysmal atrial fibrillation in stroke/tia patients. Stroke Res Treat. 2013;2013:840265.

12. Kishore A, Vail A, Majid A, et al. Detection of atrial fibrillation after ischemic stroke or transient ischemic attack: a systematic review and meta-analysis. Stroke 2014;45(2):520-526.

13. Sanna T, Diener HC, Passman RS, et al. Crystal AF Investigators Cryptogenic stroke and underlying atrial fibrillation. $N$ Engl $J$ Med. 2014;370(26):2478-2486

14. Seet RCS, Friedman PA, Rabinstein AA. Prolonged rhythm monitoring for the detection of occult paroxysmal atrial fibrillation in ischemic stroke of unknown cause. Circulation. 2011;124(4):477-486.

15. Zimetbaum P, Waks WJ, Ellis RE. Advances in Arrhythmia and Electrophysiology. Role of Atrial Fibrillation Burden in Assessing Thromboembolic Risk. Circulation: Arrhythmia and Electrophysiology. 2014;7(6):1223-1229.

16. January TC, Wann S, Alpert SJ, et al. 2014 AHA/ACC/HRS Guideline for the Management of Patients with Atrial Fibrillation.: A Report of the American College of Cardiology/American Heart Association Task Force on Practice Guidelines and the Heart Rhythm Society. Circulation. 2014;130(23):2071-2104.

17. Healy SJ, Connoly JS, Gold RM, et al. Subclinical Atrial Fibrillation and the Risk of Stroke. N Engl J Med. 2012;366(6):120-129.

18. Pipilis A, Farmakis D, Kaliambakos S, et al. Anticoagulant therapy is prescribed less often in paroxysmal atrial fibrillation regardless of thromboembolic risk: results from the Registry of Atrial Fibrillation To Investigate New Guidelines (RAFTING).Int $J$ Cardiol. 2014;175 (3):569-570

19. Cha MJ, Kim YD, Nam HS, et al. Stroke mechanisms in patients with non-valvular atrial fibrillation according to the CHADS2 and CHA2 DS2 -VASc scores. Eur J Neurol. 2012;19(3):473-479.

20. Saver LJ. Clinical Practice. Cryptogenic Stroke. $N$ Engl $J$ Med. 2016;374(21):2065-2074

21. Botto GL, Padeletti L, Santini M, et al. Presence and duration of atrial fibrillation detected by continuous monitoring: crucial implications for the risk of thromboembolic events. $J$ Cardiovasc Electrophysiol. 2009;20(3):241-248.

22. Tanne D, Molshatzki N, Merzeliak O, et al. Anemia status, hemoglobin concentration and outcome after acute stroke: a cohort study. $B M C$ Neurol. 2010;10:22 